\title{
Über einen positiven Spinalganglien- und Rückenmarksbefund bei einem Fall von Lupus erythematodes mit akutem Nachschub.
}

Von

Dr. Moriz Biach.

Mit Rücksicht auf die bisher seltenen Publikationen von Rückenmarks- und Spinalganglienuntersuchungen bei Hauterkrankungen dürften vielleicht die nachfolgenden Befunde einiges Interesse erwecken.

Klinik und histologische Hautbefunde dieses Falles werden an anderer Stelle veröffentlicht (v. Zumbus ch-Reitmann).

Das Material verdanke ich der Liebenswürdigkeit der Herren Hofrat Weichselbaum und Professor Riehl.

Die Präparate wurden in Formol fixiert und in Alkohol gehärtet. Die Einbettung wurde in Celloidin vorgenommen, die Färbung wurde zum Teil mit Hämalaun-Eosin, zum Teil nach Nissl und Weigert durchgeführt. Die Schnitte wurden nicht in Serien angefertigt, da es sich für meine Zwecke als unnötig erwies. Nachfolgend die erhobenen Befunde:

Im Querschnitt erscheinen die Ganglien in toto verkleinert, ihre Kapsel ist nur sehr mäßig verbreitert; im Innern ist das Bindegewebe ziemlich stark vermehrt und es fällt insbesonders auf, daß die Ganglienzellen eine beträchtliche Verminderung an Zahl erfahren haben. 
Wenn man nach der Ursache dieser Veränderung sucht, so sieht man einzelne Zelltorsi von einer großen Menge Kapselendothelzellen umgeben, wie denn überhaupt die Wucherung der Endothelzellen stark hervortritt. Manche der erkrankten atrophischen Ganglienzellen sind von einem dreifachen Ring von Kapselendothel umgeben. Auch echte Neuronophagie, Eindringen von Endothelien in die Ganglienzellen, ist zu sehen.

Die Ganglienzellen selbst zeigen keinerlei axonale Degeneration, das Einzige, was auffällig ist, ist die Vermehrung des braunen und auch hellgelben Pigments; man kann demnach den Prozeß in der Zelle selbst als eine einfache Atrophie oder Pigmentatrophie bezeichnen.

Der Zellkern zeigt die verschiedenen Stadien der homogenen Kernschrumpfung, insbesondere die schwereren Stadien bis zu kompletter Zerstörung des Kerns.

Auch das perineurale Gewebe der eintretenden respektive austretenden Wurzeln erscheint verdickt und zellreicher.

Diese genannten Veränderungen finden sich in den verschiedenen darauf untersuchten Ganglien vor, nur quantitativ besteht ein gewisser Unterschied, während qualitativ die Vorgänge die gleichen sind.

Im $\mathrm{N}$ is s l-Präparat kann man nur eine Bestätigung des eben Geschilderten sehen, die Tigroidfärbung tritt an den noch nicht völlig zerstörten Zellen deutlich hervor.

Von einem Infiltrat, wie es sich bei zosterähnlichen Veränderungen des Ganglion findet, ist nicht die Rede.

In der Medulla spinalis finden sich zum Unterschied von den Spinalganglienveränderungen Zeichen einer akuten Erkrankung; am deutlichsten ausgesprochen in den Meningen, wo sich eine leichte Infiltration rom Charakter der Meningitis serosa erkennen läßt. Weiters findet sich im Lendenmark ein mikroskopisch kleiner Herd im rechten Vorderseitenstrang vom Charakter der myel. Herde. Im Halsmark dagegen zeigen sich reichliche kleinste Blutungen sowohl in der grauen als in der weißen Substanz.

Parallel mit diesen interstitiellen Veränderungen geht die Degeneration der Vorderhornzellen, die jedoch keineswegs beträchtliche Grade annimmt und deutlich axonaler Natur ist. 
Faßt man nun die Ergebnisse dieser Untersuchung zusammen, so ist es von vornherein ausgeschlossen, die eben geschilderten Veränderungen des Rückenmarks etwa mit dem Lupus erythematosus akutus in Zusammenhang zu bringen.

Die Veränderungen sind hier akutester Natur und stehen im allerersten Beginn, es sind die Veränderungen einer leichten Meningomyelitis, sowie sie etwa von Sawada bei den verschiedensten Infektionskrankheiten beschrieben wurden; die im Rückenmark gefundenen Hämorrhagien sprechen nicht dagegen, wenn man berïcksichtigt, $\mathrm{da} \beta$ auch diese von Marburg bei verschiedenen Infektionen gefunden wurden.

Etwas anderes ist es mit den Veränderungen der Spinalganglien; hier handelt es sich um einen chronischen atrophischen ProzeB, bei dem es den Anschein bat, als ob er seinen Ausgangspunkt von den Spinalganglienzellen nähme.

Trotzdem die Patientin erst 22 Jahre alt war, zeigte sich eine weit rorgeschrittene, gewöhnliche oder Pigmentatrophie der Zellen und parallel mit dieser Parenchymdegeneration eine Wucherung der Kapselendothelien und des Bindegewebes; gerade der Umstand, daß jegliche Entzündungserscheinung fehlt, spricht für eine derartige Entstehungsweise.

Wenn wir die erhaltenen Resultate kurz resümieren, so ergibt sich, daß wir bei einer 22jäbrigen Kranken, die an einem chronischen Lupus erythematosus mit akutem Nachschub gelitten hat, schwere Veränderungen an den Ganglienzellen im Sinne einer Atrophie derselben gefunden haben und im Rückenmark gleichfalls Veränderungen vom Charakter der Meningitis serosa.

Es erwächst für uns die Frage: welcher Zusammenhang besteht zwischen diesen Befunden und der bezeichneten Hauterkrankung?

Erwägt man, dab in den Fällen von Herpes zoster, bei denen eine Ganglienerkrankung gefunden wurde, diese ganz anderer Natur war als die hier erhobene, indem eine echte Entzündung der Ganglienzellen besteht, und da man wohl berechtigt ist anzunehmen, $\mathrm{da} \beta$ zwischen der beim Zoster vorhandenen Hauterkrankung und der Erkrankung der Ganglien ein inniger Zusammenhang besteht, so ist $\operatorname{der}$ Schluß nicht 
unberechtigt, überhaupt an Beziehungen zwischen Ganglienund Hauterkrankungen zu denken. Auch unsere Befunde scheinen mir mit einer gewissen Sicherheit für diesen $\mathrm{Zu}$ sammenhang zu sprechen, indem man in diesem Alter an anderen Erkrankungen - mit Ausnahme von akuten Infektionskrankheiten - verstorbenen Patienten solche Befunde an Ganglien nicht zu erheben imstande ist.

Die vorhandene Atrophie kommt nur in sehr hohem Alter vor, im jugendlichen Alter bedeutet sie einen pathologischen Vorgang, der sich in längerer oder kürzerer Zeit an den Ganglienzellen abspielt.

Inwiefern gerade die Hautveränderungen diese Atrophie der Ganglienzellen verursachen können, ist schwer zu sagen, trotzdem aber glauben wir, da sich ja auch bei anderen Hauterkrankungen mit einer gewissen Konstanz solche Veränderungen an den Ganglienzellen vorfanden, daß gerade Hauterkrankungen mit einer gewissen Vorliebe von Ganglienerkrankungen begleitet werden.

Ich werde versuchen das Material noch weiterhin zu vervollständigen, indem ich weitere Untersuchungen von Rückenmark und Spinalganglien nach Hauterkrankungen akuter und chronischer Natur vorzunehmen gedenke. 